\title{
Energetic Efficiency of Infant Formulae: A Review
}

\author{
Manja Fleddermann Hans Demmelmair Berthold Koletzko \\ Dr. von Hauner Children's Hospital, University of Munich Medical Center, Munich, Germany
}

\section{Key Words}

Energy utilization · Energy intake · Formula feeding ·

Growth · Infant formula

\begin{abstract}
Breast-fed and formula-fed infants differ in terms of nutrient intake, growth, and metabolic and endocrine responses. The energetic efficiency, i.e. the weight or length gain per 100 kcal of energy intake, of breast-fed infants is about $11 \%$ higher than the energetic efficiency of formula-fed infants. Only limited data is available on the influence of formula composition on the energetic efficiency of infant formulae. We conducted a review of controlled trials to identify the impact of the macronutrient composition of infant formulae on energetic efficiency. An electronic literature search was conducted in February 2014. Intervention trials that investigated the effect of an infant formula with a modified macronutrient composition and reported the weight, length, and nutritional intake of apparently healthy, term, fully formula-fed infants with a normal weight were included. Thirteen trials met the inclusion criteria. The results showed no effect of the total content of energy, carbohydrate, protein, or fat on energetic efficiency. In contrast, small increasing effects of higher glycemic carbohydrates on energetic efficiency were identified. Improved fat absorption via the use of palmitic acid at the sn-2 ester position of triacylglycerol increased the energetic
\end{abstract}

\section{KARGER}

E-Mail karger@karger.com

www.karger.com/anm efficiency by $11 \%$. The quality of formula protein, specifically an increased whey-to-casein ratio, an increased a-lactalbumin content, or a higher tryptophan content increased the energetic efficiency by about $13 \%$. We conclude that fat absorption and protein quality have the potential to modulate energetic efficiency and may contribute to the observed differences in growth and metabolism between breast-fed and formula-fed infants.

(c) 2014 S. Karger AG, Basel

\section{Introduction}

Compared to breast-fed (BF) infants, the energy requirements of formula-fed (FF) infants are about $15 \%$ higher at the age of 1 month and about $7 \%$ higher at the age of 6 months [1]. Observational data indicate that energetic efficiency (EE; growth-weight gain or length gain per $100 \mathrm{kcal}$ ) is $11 \%$ higher in $\mathrm{BF}$ infants than in $\mathrm{FF}$ infants $[2,3]$. Limited data is available on the relationship between the composition of infant formulae and their corresponding EE for growth. However, FF infants consume up to $26 \%$ more energy and show higher mean weight gains than BF infants [3]. This higher early weight gain velocity has been associated with an increased risk of obesity and type 2 diabetes in later life [4-8]. 
We observed differences in EE in a study on 213 healthy term infants who were randomized to either a reduced-protein infant formula with a higher $\alpha$-lactalbumin (ALAB) content and added long-chain polyunsaturated fatty acids (LC-PUFA) or a control formula (CF) with a standard protein content without LC-PUFA until the age of 120 days [9]. The mean daily weight gain was tendentially higher at $30.2 \mathrm{~g}$ in the reduced-protein group than in the control group ( $28.3 \mathrm{~g}$ ). Infants in the reducedprotein group consumed significantly less energy during the study period ( $490 \mathrm{vs.} 531 \mathrm{kcal} /$ day). Thus, the EE for weight was about $12 \%$ higher in infants who received the reduced-protein formula with ALAB and LC-PUFA, which we considered to be induced by different dietary protein and amino acid compositions [9].

This finding prompted us to review the available data on the effects of macronutrients of infant formulae on EE in fully FF infants during the first months of life. We aimed to identify the relationship of infant formula composition with EE for growth and searched for effects of macronutrient contents and of modified protein, fat, and carbohydrate sources.

\section{Methods}

A structured electronic literature search was carried out on February 7, 2014, on PubMed.gov. The search term was: [infant formula] not [preterm or premature] and [Clinical Trial] and [humans]. The studies included in the review met the following criteria: (1) the effect of an infant formula was investigated, (2) weight and length and energy intake were evaluated, (3) an FF control group was included, and (4) participants were apparently healthy infants born at term ( $\geq 37$ weeks of gestation) with a normal weight $(>2,500 \mathrm{~g}$ at birth or a weight above the 5 th percentile of WHO growth charts) and were fully FF (contribution of other foods was $<25 \%$ of the energy intake).

Full-text articles which met these inclusion criteria were extracted into an EndNote database (EndNote X5; Thomson Reuters, Philadelphia, Pa., USA). Information on methods, populations, interventions, growth, and nutritional intakes was collected. If the mean weight, length gain, and dietary intake were not reported in grams/ day, millimeters/day, and kilocalories/day, respectively, they were calculated using reported data. If the growth or dietary intake could not be calculated from reported data, the study was excluded from analysis. Additionally, the study of Fleddermann et al. [9] was considered in the analysis and used as an additional reference.

Growth and energy intakes were compared between intervention and control groups for all included studies. When the dietary intake was reported in kilocalories/kilogram of body weight, the mean dietary energy intake was calculated using the absolute weight at assessment of the dietary intake. Based on Fleddermann et al. [9], the EE was calculated as the ratio of weight or length gain (expressed as $\mathrm{g} /$ day or $\mathrm{mm} /$ day) and the average energy intake per day during a study. EE was expressed as grams/100 kilocalories for weight or as millimeters/100 kilocalories for length.

\section{Results}

\section{Selection of Eligible Papers}

The literature search yielded 808 references. Of those 808 studies, 620 were excluded on the basis of titles and abstracts not fulfilling our inclusion criteria, while 188 were identified as potentially eligible (fig. 1). In total, 94 studies with a focus on the macronutrient composition of infant formulae were obtained as full-text articles. After a detailed evaluation, 13 studies of different population groups met the inclusion criteria and were included in the analysis. The main reason for exclusion was inadequate reporting of the energy intake or growth.

\section{Baseline Characteristics and Results of the Included Trials}

The key characteristics of the included trials are presented in table 1 . All studies assessed growth and energy intake from birth or the first month of life until the ages of 3-6 months. All but 2 studies included infants of both sexes, while Fomon et al. [10] and Carnielli et al. [11] enrolled male infants only. Ten of 14 studies aimed to investigate the suitability and safety of the formulae with a focus on protein and carbohydrate contents [2, 10, 12-19], while studies focused on fat composition aimed to investigate fat or mineral balances $[11,20,21]$. Schmelzle et al. [17] and Lasekan et al. [19] studied modified carbohydrate components in formulae. The studies of Koo et al. [20], Kennedy et al. [21], and Carnielli et al. [11] investigated formulae with modified fat contents. Most of the included studies focused on the protein component $[2$, $10,12-17]$.

In total, the studies evaluated data of 1,561 participants. The duration of the interventions varied from 35 days to 6 months. Further details on the population characteristics, compositions of formulae, and available data of the included studies are shown in table 1.

\section{Growth and Energy Intake}

The observed weight gains were 22.5-35.9 g/day, and length gains varied from 0.87 to $1.59 \mathrm{~mm} /$ day (table 2). The anthropometric gains differed between the different study populations and tended to be higher in smaller infants. The highest anthropometric gains (31.7-35.9 g/day or $1.27-1.29 \mathrm{~mm} /$ day) were observed in infants who were 8-12 weeks old at the study end, compared to lower gains in infants aged 6 months at the study end (22.5-24.4 g/ day or $0.87-0.99 \mathrm{~mm} /$ day).

The energy intake for study formulae varied between 395 and $718 \mathrm{kcal} /$ day (table 2). The average energy intake 
Fig. 1. Articles identified, screened, assessed, and excluded at various stages of this analysis.

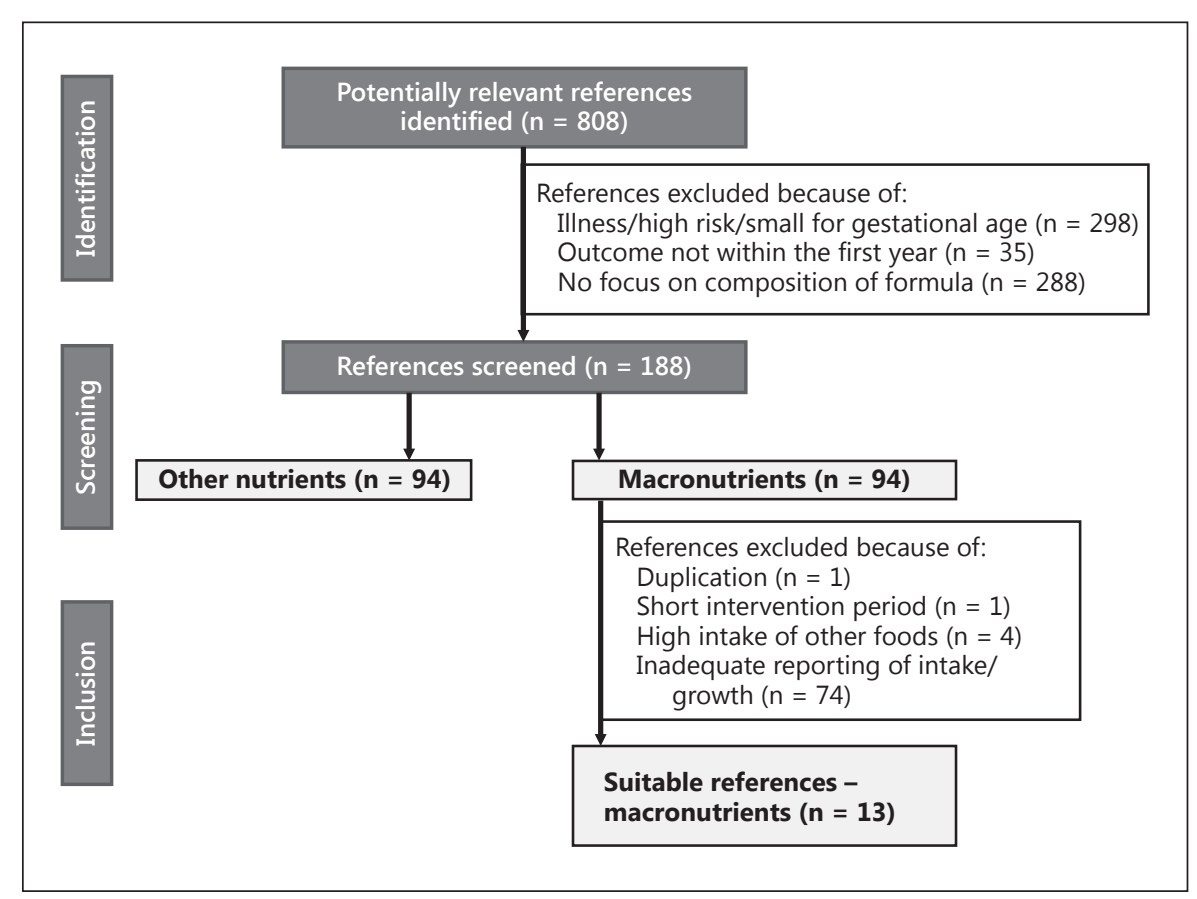

Table 1. Summary of the main characteristics and main results of the included trials

\begin{tabular}{|c|c|c|c|c|c|}
\hline Reference & Population characteristics & IF 2 & CF & Age & Outcomes \\
\hline $\begin{array}{l}\text { Fleddermann } \\
\text { et al. [9] }\end{array}$ & $\begin{array}{l}\text { Apparently healthy term } \\
\text { singletons, } 37-41 \text { weeks } \\
\text { of gestation, birth weight } \\
\text { between the } 3 \text { rd and } 97 \text { th } \\
\text { percentiles of Euro growth } \\
\text { charts, } \mathrm{n}=107 \text { (IF), } \mathrm{n}= \\
106(\mathrm{CF})\end{array}$ & $\begin{array}{l}67 \mathrm{kcal}, 1.3 \mathrm{~g} \text { protein } \\
\text { (higher ALAB content, free } \\
\text { Trp and phenylalanine), } \\
3.6 \mathrm{~g} \text { fat ( } 10.7 \mathrm{mg} \\
\text { arachidonic and } \\
\text { docosahexaenoic acid), } \\
7.5 \mathrm{~g} \text { carbohydrates }\end{array}$ & $\begin{array}{l}67 \mathrm{kcal}, 1.5 \mathrm{~g} \text { protein, } \\
3.3 \mathrm{~g} \text { fat (no LC-PUFA), } \\
7.8 \mathrm{~g} \text { carbohydrates }\end{array}$ & $\begin{array}{l}\text { Birth to } \\
120 \text { days }\end{array}$ & $\begin{array}{l}\text { Weight gain } \\
\text { (g/day), length } \\
\text { gain (cm/day), } \\
\text { energy intake } \\
\text { (kcal/day) }\end{array}$ \\
\hline $\begin{array}{l}\text { Fomon et al. } \\
{[10]}\end{array}$ & $\begin{array}{l}\text { Male term infants, birth } \\
\text { weight }>2,500 \mathrm{~g}, \mathrm{n}=13 \\
(\mathrm{CF}), \mathrm{n}=15 \text { (IF) }\end{array}$ & $\begin{array}{l}67 \mathrm{kcal}, 0.8 \mathrm{~g} \text { protein, } 3.75 \mathrm{~g} \\
\text { fat, } 7.58 \mathrm{~g} \text { carbohydrates }\end{array}$ & $\begin{array}{l}67 \mathrm{kcal}, 1.14 \mathrm{~g} \text { protein, } \\
3.75 \mathrm{~g} \text { fat, } 7.29 \mathrm{~g} \\
\text { carbohydrates }\end{array}$ & $\begin{array}{l}\text { Birth to } \\
112 \text { days }\end{array}$ & $\begin{array}{l}\text { Weight gain } \\
\text { (g/day), length } \\
\text { gain }(\mathrm{mm} / \text { day), } \\
\text { energy intake } \\
(\mathrm{kcal} / \mathrm{kg} / \text { day })\end{array}$ \\
\hline $\begin{array}{l}\text { Turck et al. } \\
{[12]}\end{array}$ & $\begin{array}{l}\text { Healthy term newborns, } \\
37-42 \text { weeks of gestation, } \\
\text { birth weight } 2,500- \\
4,200 \mathrm{~g}, \mathrm{n}=51(\mathrm{IF}) \\
\mathrm{n}=50(\mathrm{CF})\end{array}$ & $\begin{array}{l}67 \mathrm{kcal}, 1.2 \mathrm{~g} \text { protein }(30 \% \\
\text { casein } / 70 \% \text { whey), } 7.5 \mathrm{~g} \\
\text { carbohydrates ( } 85 \% \\
\text { lactose } / 15 \% \text { maltodextrin), } \\
3.6 \mathrm{~g} \text { fat }\end{array}$ & $\begin{array}{l}67 \mathrm{kcal}, 1.7 \mathrm{~g} \text { protein } \\
\text { ( } 70 \% \text { casein } / 30 \% \text { whey), } \\
7.8 \mathrm{~g} \text { carbohydrates } \\
\text { (70\% lactose } / 30 \% \\
\text { maltodextrin), } 3.2 \mathrm{~g} \text { fat }\end{array}$ & $\begin{array}{l}\text { Birth to } \\
120 \text { days }\end{array}$ & $\begin{array}{l}\text { Weight gain } \\
\text { (g/day), length } \\
\text { gain }(\mathrm{mm} / \text { day }) \text {, } \\
\text { energy intake } \\
(\mathrm{kcal} / \mathrm{kg} / \text { day })\end{array}$ \\
\hline $\begin{array}{l}\text { Rozé et al. } \\
{[13]}\end{array}$ & $\begin{array}{l}>37 \text { weeks of gestation, } \\
\text { without human milk } \\
\text { before inclusion, } n=43 \\
(\mathrm{IF}), \mathrm{n}=45(\mathrm{CF})\end{array}$ & $\begin{array}{l}68 \mathrm{kcal}, 1.4 \mathrm{~g} \text { protein }(0.3 \mathrm{~g} \\
\mathrm{ALAB}, 60 \% \text { whey } / 40 \% \\
\text { casein), } 7.6 \mathrm{~g} \text { carbohydrates, } \\
3.5 \mathrm{~g} \text { fat }(12.6 \mathrm{mg} \\
\text { arachidonic acid, } 7 \mathrm{mg} \\
\text { docosahexaenoic acid) plus } \\
\text { different symbiotics }\end{array}$ & $\begin{array}{l}72 \mathrm{kcal}, 1.5 \mathrm{~g} \text { protein } \\
\text { ( } 50 \% \text { whey/50\% casein), } \\
8.9 \mathrm{~g} \text { carbohydrates, } \\
3.4 \mathrm{~g} \text { fat (no arachidonic } \\
\text { acid or docosahexaenoic } \\
\text { acid), no symbiotics }\end{array}$ & $\begin{array}{l}\text { Birth to } \\
6 \text { months }\end{array}$ & $\begin{array}{l}\text { Weight, length } \\
\text { (absolute at } \\
\text { baseline and } 6 \\
\text { months), energy } \\
\text { intake (kcal/day) }\end{array}$ \\
\hline $\begin{array}{l}\text { Davis et al. } \\
{[14]}\end{array}$ & $\begin{array}{l}\text { Healthy term infants, } \\
\text { weight-for-length at birth } \\
\text { (10th to 90th percentiles } \\
\text { of the Centers for Disease } \\
\text { Control and Prevention), } \\
\mathrm{n}=64 \text { for each group }\end{array}$ & $\begin{array}{l}67 \mathrm{kcal}, 1.4 \mathrm{~g} \text { protein } \\
(0.22 \mathrm{~g} \text { ALAB }), 7.3 \mathrm{~g} \\
\text { carbohydrates, } 3.6 \mathrm{~g} \text { fat }\end{array}$ & $\begin{array}{l}67 \mathrm{kcal}, 1.5 \mathrm{~g} \text { protein } \\
(0.13 \mathrm{~g} \text { ALAB }) \\
7.3 \mathrm{~g} \text { carbohydrates, } \\
3.6 \mathrm{~g} \text { fat }\end{array}$ & $\begin{array}{l}\text { Birth to } \\
8 \text { weeks }\end{array}$ & $\begin{array}{l}\text { Weight gain } \\
\text { (g/day), length } \\
\text { gain }(\mathrm{mm} / \text { day), } \\
\text { energy intake } \\
\text { (kcal/day) }\end{array}$ \\
\hline
\end{tabular}


Table 1 (continued)

\begin{tabular}{|c|c|c|c|c|c|c|}
\hline Reference & Population characteristics & IF 1 & IF 2 & $\mathrm{CF}$ & Age & Outcomes \\
\hline $\begin{array}{l}\text { Räihä et al. } \\
{[15]}\end{array}$ & $\begin{array}{l}\text { Healthy newborns, } 37-42 \\
\text { weeks of gestation, birth } \\
\text { weight } 2,501-4,500 \mathrm{~g}, \mathrm{n}= \\
29(\mathrm{IF}), \mathrm{n}=27(\mathrm{IF} 2), \mathrm{n}= \\
29(\mathrm{CF})\end{array}$ & $\begin{array}{l}66.3 \mathrm{kcal}, 1.26 \text { g protein } \\
\text { (modified sweet whey, } \\
70 \% \text { whey } / 30 \% \text { casein), } \\
\text { higher lactose content }\end{array}$ & $\begin{array}{l}65.9 \mathrm{kcal}, 1.26 \mathrm{~g} \\
\text { protein (acid } \\
\text { whey, } 70 \% \\
\text { whey } / 30 \% \text { casein), } \\
\text { higher lactose } \\
\text { content }\end{array}$ & $\begin{array}{l}65.6 \mathrm{kcal}, 1.51 \mathrm{~g} \text { protein } \\
(60 \% \text { whey } / 40 \% \text { casein })\end{array}$ & $\begin{array}{l}\text { Birth to } \\
120 \text { days }\end{array}$ & $\begin{array}{l}\text { Weight gain } \\
\text { (g/day), length } \\
\text { gain (mm/day), } \\
\text { energy intake } \\
\text { (kcal/kg/day) }\end{array}$ \\
\hline $\begin{array}{l}\text { Vandenplas } \\
\text { et al. [16] }\end{array}$ & $\begin{array}{l}\text { Healthy newborns, } \\
\text { no infants with atopic } \\
\text { disease, } n=25 \text { (IF), } \\
n=20(\mathrm{CF})\end{array}$ & $\begin{array}{l}67 \mathrm{kcal}, 1.5 \mathrm{~g} \text { protein }(100 \% \\
\text { whey protein hydrolysate), } \\
6.9 \mathrm{~g} \text { carbohydrates ( } 38 \% \\
\text { lactose } / 62 \% \text { maltose plus } \\
\text { glucose polymers), } 3.6 \mathrm{~g} \text { fat }\end{array}$ & & $\begin{array}{l}66 \mathrm{kcal}, 1.4 \mathrm{~g} \text { protein } \\
(60 \% \text { whey/40\% casein), } \\
7.1 \mathrm{~g} \text { carbohydrates } \\
(100 \% \text { lactose }), 3.6 \mathrm{~g} \text { fat }\end{array}$ & $\begin{array}{l}\text { Birth to } \\
3 \text { months }\end{array}$ & $\begin{array}{l}\text { Weight gain } \\
\text { (g/day), length } \\
\text { gain (mm/day), } \\
\text { energy intake } \\
\text { (kcal/day) }\end{array}$ \\
\hline $\begin{array}{l}\text { Hanning } \\
\text { et al. [2] }\end{array}$ & $\begin{array}{l}>37 \text { weeks of gestation, } \\
\text { appropriate weight for } \\
\text { gestational age, } n=73 \\
(\mathrm{IF}), \mathrm{n}=76(\mathrm{CF})\end{array}$ & $\begin{array}{l}73.3 \mathrm{kcal}, 1.33 \text { g protein } \\
(40 \% \text { whey } / 60 \% \text { casein, } \\
\text { plus Trp), unknown } \\
\text { carbohydrate and fat } \\
\text { contents }\end{array}$ & & $\begin{array}{l}66.9 \mathrm{kcal}, 1.59 \text { g protein } \\
(60 \% \text { whey } / 40 \% \text { casein, } \\
\text { no extra Trp), unknown } \\
\text { carbohydrate and fat } \\
\text { contents }\end{array}$ & $\begin{array}{l}4-12 \\
\text { weeks }\end{array}$ & $\begin{array}{l}\text { Weight gain } \\
\text { (g/day), length } \\
\text { gain (mm/day), } \\
\text { energy intake } \\
\text { (kcal/day) }\end{array}$ \\
\hline $\begin{array}{l}\text { Schmelzle } \\
\text { et al. [17] }\end{array}$ & $\begin{array}{l}\text { Gestational age } 37-42 \\
\text { weeks, birth weight } \\
\text { ( } 10 \text { th to } 90 \text { th percentiles), } \\
\text { singleton healthy babies, } \\
\text { mothers without } \\
\text { significant illness or } \\
\text { disabilities, } \mathrm{n}=49 \text { (IF), } \\
\mathrm{n}=52(\mathrm{CF})\end{array}$ & $\begin{array}{l}70 \mathrm{kcal}, 1.7 \mathrm{~g} \text { protein } \\
\text { ( } 100 \% \text { hydrolyzed } \\
\text { whey protein), } 8.4 \mathrm{~g} \\
\text { carbohydrates } \\
\text { ( } 34 \% \text { lactose } / 48 \% \\
\text { maltodextrin } / 18 \% \text { starch), } \\
3.3 \mathrm{~g} \text { fat, } 0.8 \text { g prebiotics }\end{array}$ & & $\begin{array}{l}67 \mathrm{kcal}, 1.5 \mathrm{~g} \text { protein } \\
(60 \% \text { whey } / 40 \% \text { casein }), \\
7.2 \mathrm{~g} \text { carbohydrates } \\
\text { ( } 100 \% \text { lactose), } 3.6 \mathrm{~g} \text { fat, } \\
\text { no prebiotics }\end{array}$ & $\begin{array}{l}2-12 \\
\text { weeks }\end{array}$ & $\begin{array}{l}\text { Weight gain } \\
\text { (g/day), length } \\
\text { gain (mm/day), } \\
\text { energy intake } \\
\text { (kcal/kg/day) }\end{array}$ \\
\hline $\begin{array}{l}\text { Heubi et al. } \\
{[18]}\end{array}$ & $\begin{array}{l}\text { Healthy term infants, } \\
\text { gestational age } 38-42 \\
\text { weeks, } n=70(\mathrm{IF}), \mathrm{n}=67 \\
(\mathrm{CF})\end{array}$ & $\begin{array}{l}67.2 \text { kcal, } 1.5 \text { g protein, } \\
7.2 \text { g carbohydrates }(75 \% \\
\text { maltodextrin } / 25 \% \text { sucrose }) \text {, } \\
3.6 \text { g fat }\end{array}$ & & $\begin{array}{l}67.2 \mathrm{kcal}, 1.5 \mathrm{~g} \text { protein, } \\
7.2 \mathrm{~g} \text { carbohydrates } \\
(100 \% \text { lactose }), 3.6 \mathrm{~g} \text { fat }\end{array}$ & $\begin{array}{l}\text { Birth to } \\
3 \text { months }\end{array}$ & $\begin{array}{l}\text { Weight, length } \\
\text { (absolute at } \\
\text { baseline and } 12 \\
\text { weeks), energy } \\
\text { intake (kcal/kg/ } \\
\text { day) }\end{array}$ \\
\hline $\begin{array}{l}\text { Lasekan et al. } \\
{[19]}\end{array}$ & $\begin{array}{l}\text { Healthy, full-term } \\
\text { singletons, gestational } \\
\text { weeks } 37-42 \text {, weight } \\
\text { ( } 5 \text { th and 95th percentiles } \\
\text { of the National Center for } \\
\text { Health Statistics), } \mathrm{n}=63 \\
(\mathrm{IF}), \mathrm{n}=65(\mathrm{CF})\end{array}$ & $\begin{array}{l}67.6 \mathrm{kcal}, 1.4 \mathrm{~g} \text { protein, } 7.23 \\
\text { g carbohydrates } \\
\text { ( } 55 \% \text { corn syrup } / 45 \% \\
\text { sucrose), } 3.65 \mathrm{~g} \text { fat }\end{array}$ & & $\begin{array}{l}67.6 \mathrm{kcal}, 1.4 \mathrm{~g} \text { protein, } \\
7.23 \mathrm{~g} \text { carbohydrates } \\
(100 \% \text { lactose }), 3.65 \mathrm{~g} \text { fat }\end{array}$ & $\begin{array}{l}\text { Birth to } \\
112 \text { days }\end{array}$ & $\begin{array}{l}\text { Weight gain } \\
\text { (g/day), absolute } \\
\text { length, energy } \\
\text { intake (kcal/day) }\end{array}$ \\
\hline $\begin{array}{l}\text { Koo et al. } \\
{[20]}\end{array}$ & $\begin{array}{l}\text { Healthy singletons, } \\
<2 \text { weeks, gestational age } \\
37-42 \text { weeks, } n=63 \text { (IF), } \\
\mathrm{n}=65(\mathrm{CF})\end{array}$ & $\begin{array}{l}67.6 \mathrm{kcal}, 1.42 \mathrm{~g} \text { protein } \\
\text { (nonfat milk protein, } \\
\text { reduced mineral whey), } \\
7.37 \mathrm{~g} \text { carbohydrates, } \\
3.58 \mathrm{~g} \text { fat }(45 \% \text { palm } / \text { palm } \\
\text { olein) }\end{array}$ & & $\begin{array}{l}67.6 \mathrm{kcal}, 1.4 \mathrm{~g} \text { protein } \\
\text { (nonfat milk protein, } \\
\text { whey protein } \\
\text { concentrate), } 7.3 \mathrm{~g} \\
\text { carbohydrates, } 3.65 \mathrm{~g} \text { fat } \\
\text { (without palm olein) }\end{array}$ & $\begin{array}{l}6 \text { days to } \\
6 \text { months }\end{array}$ & $\begin{array}{l}\text { Weight, length } \\
\text { (absolute), } \\
\text { energy intake } \\
\text { (kcal/day) }\end{array}$ \\
\hline $\begin{array}{l}\text { Kennedy } \\
\text { et al. [21] }\end{array}$ & $\begin{array}{l}\geq 37 \text { weeks of gestation, } \\
\text { birth weight }>5 \text { th } \\
\text { percentile, } n=100(\mathrm{IF}), \\
\mathrm{n}=103(\mathrm{CF})\end{array}$ & $\begin{array}{l}73 \mathrm{kcal}, 1.6 \mathrm{~g} \text { protein, } \\
7.1 \mathrm{~g} \text { carbohydrates, } \\
4.2 \mathrm{~g} \text { fat ( } 50 \% \text { palmitate at } \\
\text { the sn- } 2 \text { position) }\end{array}$ & & $\begin{array}{l}70 \text { kcal, } 1.6 \text { g protein, } \\
7.1 \text { g carbohydrates, } \\
3.9 \text { g fat ( } 12 \% \text { palmitate } \\
\text { at the sn- } 2 \text { position) }\end{array}$ & $\begin{array}{l}8 \text { days to } \\
12 \text { weeks }\end{array}$ & $\begin{array}{l}\text { Weight, length } \\
\text { (absolute at } \\
\text { randomization } \\
\text { and } 12 \text { weeks), } \\
\text { energy intake } \\
\text { (kcal/day) }\end{array}$ \\
\hline $\begin{array}{l}\text { Carnielli } \\
\text { et al. [11] }\end{array}$ & $\begin{array}{l}\text { Healthy term males, free } \\
\text { of manifest disease, } n=9 \\
\text { for each group }\end{array}$ & $\begin{array}{l}66 \mathrm{kcal}, 1.4 \mathrm{~g} \text { protein, } \\
3.6 \mathrm{~g} \text { fat }(23.9 \% \text { palmitic } \\
\text { acid, } 66 \% \text { of which } \\
\text { esterified to the sn- } 2 \\
\text { position), unknown } \\
\text { carbohydrates }\end{array}$ & $\begin{array}{l}66 \mathrm{kcal}, 1.4 \mathrm{~g} \\
\text { protein, } 3.6 \mathrm{~g} \text { fat } \\
\text { ( } 24 \% \text { palmitic } \\
\text { acid, } 39 \% \text { of which } \\
\text { esterified to the } \\
\text { sn-2 position), } \\
\text { unknown } \\
\text { carbohydrates }\end{array}$ & $\begin{array}{l}66 \mathrm{kcal}, 1.4 \mathrm{~g} \text { protein, } \\
3.6 \mathrm{~g} \text { fat ( } 19.9 \% \text { palmitic } \\
\text { acids, } 13 \% \text { of which } \\
\text { esterified to the sn- } 2 \\
\text { position), unknown } \\
\text { carbohydrates }\end{array}$ & $\begin{array}{l}\text { Birth to } \\
5 \text { weeks }\end{array}$ & $\begin{array}{l}\text { Weight, length } \\
\text { (absolute at birth } \\
\text { and } 1 \text { month), } \\
\text { formula intake } \\
(\mathrm{ml} / \mathrm{kg} / \text { day) }\end{array}$ \\
\hline
\end{tabular}


Table 2. Summary of growth, energy intake, and calculated EE

\begin{tabular}{|c|c|c|c|c|c|c|}
\hline Reference & Formula & $\begin{array}{l}\text { Weight } \\
\text { gain, } \\
\text { g/day }\end{array}$ & $\begin{array}{l}\text { Length } \\
\text { gain, } \\
\mathrm{mm} / \text { day }\end{array}$ & $\begin{array}{l}\text { Energy } \\
\text { intake, } \\
\text { kcal/day }\end{array}$ & $\begin{array}{l}\text { EE } \\
\text { for weight, } \\
\mathrm{g} / 100 \mathrm{kcal}\end{array}$ & $\begin{array}{l}\mathrm{EE} \\
\text { for length, } \\
\mathrm{mm} / 100 \mathrm{kcal}\end{array}$ \\
\hline \multirow[t]{2}{*}{ Fleddermann et al. [9] } & IF & 30.2 & 1.06 & 490 & 6.45 & 0.22 \\
\hline & $\mathrm{CF}$ & 28.3 & 0.99 & 531 & 5.67 & 0.19 \\
\hline \multirow[t]{2}{*}{ Fomon et al. [10] } & IF & 29.3 & 1.04 & 577 & 5.08 & 0.18 \\
\hline & CF & 31.3 & 1.10 & 604 & 5.19 & 0.18 \\
\hline \multirow[t]{2}{*}{ Turck et al. [12] } & IF & 29.7 & 1.12 & 477 & 6.22 & 0.23 \\
\hline & $\mathrm{CF}$ & 29.3 & 1.15 & 506 & 5.79 & 0.23 \\
\hline \multirow[t]{2}{*}{ Rozé et al. [13] } & IF & 24.7 & 0.89 & 449 & 5.51 & 0.20 \\
\hline & CF & 22.5 & 0.87 & 460 & 4.90 & 0.19 \\
\hline \multirow[t]{2}{*}{ Davis et al. [14] } & IF & 35.9 & 1.29 & 661 & 5.43 & 0.20 \\
\hline & $\mathrm{CF}$ & 35.0 & 1.31 & 718 & 4.87 & 0.18 \\
\hline \multirow[t]{3}{*}{ Räihä et al. [15] } & IF & 28.1 & 0.96 & 520 & 5.40 & 0.18 \\
\hline & IF 2 & 25.1 & 0.95 & 504 & 4.97 & 0.19 \\
\hline & $\mathrm{CF}$ & 27.8 & 1.04 & 565 & 4.92 & 0.18 \\
\hline \multirow[t]{2}{*}{ Vandenplas et al. [16] } & IF & 27.2 & 1.18 & 395 & 6.88 & 0.30 \\
\hline & $\mathrm{CF}$ & 27.2 & 1.14 & 449 & 6.06 & 0.25 \\
\hline \multirow[t]{2}{*}{ Hanning et al. [2] } & IF & 31.7 & 1.23 & 520 & 6.09 & 0.24 \\
\hline & $\mathrm{CF}$ & 30.6 & 1.27 & 583 & 5.25 & 0.22 \\
\hline \multirow[t]{2}{*}{ Schmelzle et al. [17] } & IF & 35.6 & 1.30 & 490 & 7.26 & 0.27 \\
\hline & $\mathrm{CF}$ & 32.7 & 1.20 & 510 & 6.41 & 0.24 \\
\hline \multirow[t]{2}{*}{ Heubi et al. [18] } & IF & 33.6 & 1.18 & 528 & 6.37 & 0.22 \\
\hline & $\mathrm{CF}$ & 32.7 & 1.21 & 525 & 6.23 & 0.23 \\
\hline \multirow[t]{2}{*}{ Lasekan et al. [19] } & IF & 31.1 & 1.15 & 508 & 6.12 & 0.23 \\
\hline & $\mathrm{CF}$ & 29.4 & 1.15 & 496 & 5.93 & 0.23 \\
\hline \multirow[t]{2}{*}{ Koo et al. [20] } & IF & 24.4 & 0.97 & 711 & 3.43 & 0.14 \\
\hline & $\mathrm{CF}$ & 25.5 & 0.99 & 698 & 3.65 & 0.14 \\
\hline \multirow[t]{2}{*}{ Kennedy et al. [21] } & IF & 33.2 & 1.27 & 526 & 6.31 & 0.25 \\
\hline & CF & 33.2 & 1.32 & 540 & 6.15 & 0.24 \\
\hline \multirow[t]{3}{*}{ Carnielli et al. [11] } & IF & 29.2 & 1.29 & 417 & 7.01 & 0.31 \\
\hline & IF 2 & 23.3 & 1.26 & 416 & 5.58 & 0.30 \\
\hline & $\mathrm{CF}$ & 26.6 & 1.59 & 426 & 6.24 & 0.37 \\
\hline
\end{tabular}

for the new established and modified intervention formulae (IF) was lower (up to $63 \mathrm{kcal} /$ day) than or similar (maximum $13 \mathrm{kcal} /$ day higher in 3 studies) to the energy intake provided by $\mathrm{CF}$.

\section{Calculated EE}

The EE for growth was calculated for all studies and ranged from 3.43 to $7.26 \mathrm{~g} / 100 \mathrm{kcal}$ for weight gain and from 0.14 to $0.37 \mathrm{~mm} / 100 \mathrm{kcal}$ for length gain (table 2). The largest difference in $\mathrm{EE}$ between the inter- vention and control groups of one study (EE of IF minus $\mathrm{EE}$ of $\mathrm{CF}$ ) was observed in 6 of 9 protein-focused studies $[2,9,13,14,16,17]$ and in 1 study on fat modification [11].

There were no consistent effects of the total contents of energy, protein, carbohydrates, or fat on EE. Regarding protein quality, Davis et al. [14], Fleddermann et al. [9], and Rozé et al. [13] observed an about 10-12\% higher EE for weight with ALAB-enriched formulae. A higher whey-to-casein ratio $[12,13,15,17,20]$ as well as a 
higher tryptophan (Trp) content $[2,9]$ also enhanced the EE. Modifications of the carbohydrate sources of infant formulae resulted in only modest increases in EE of $2-3 \%$ when combinations of maltodextrin, sucrose, or corn syrup and lactose were compared to lactose-only formulae [17-19]. Studies on modification of the fat source, with use of a structured triacylglycerol with preferential esterification of palmitic acid at the sn-2 position, reported an increased EE of $4-11 \%$ with a high palmitic acid content at the sn-2 position of triacylglycerols $[11,21]$.

\section{Discussion}

This review documents the effects of infant formula composition on EE. Intervention trials on infants from birth to 6 months of age were conducted on 1,561 infants. The results showed no effect of total contents of energy, protein, carbohydrates, or fat on EE, but did show effects of macronutrient quality or source.

The results on modification of the carbohydrate source showed no or only a slight impact on EE of 2$4 \%$. This small increase might be related to carbohydrates with a higher glycemic index, such as maltodextrin (glycemic index 80-105), corn syrup (glycemic index 115), sucrose (glycemic index 65), and starch (glycemic index 100), as compared to lactose (glycemic index 46), which may result in elevated blood glucose and insulin levels [22]. We have proposed that elevated insulin levels may induce enhanced infant weight gain and fat deposition and an increased later risk of adiposity and obesity [23].

The effect of palmitic acid positioning in the dietary triacylglycerol was investigated in studies on fat modification [11, 20, 21]. Palmitic acid is the most abundant saturated fatty acid in human milk triacylglycerols [24]. Use of a structured triacylglycerol with preferential esterification of palmitic acid at the sn-2 ester position instead of the sn-1,3 position increased the EE for weight by about $11 \%$ and that for length by about $16 \%$ [11]. The sn-2 ester bond is not cleaved during the digestion by pancreatic lipase, and the resulting sn-2 palmitoyl monoacylglycerol is more efficiently absorbed than free palmitic acid [11], which increases the metabolizable energy.

The major factor influencing EE that we identified is protein quality. Improved protein quality of formulae may result in a lower total energy intake via formula $[9$, 12-15] with a sufficient supply of bioavailable essential amino acids. In infant formula, Trp becomes the first limiting amino acid when the protein content is reduced and no free amino acids are added [25]. An adequate supply of Trp can be achieved via the addition of free Trp or an increase in ALAB content. As the predominant whey fraction in human milk, ALAB is the main Trp source in BF infants, and its incorporation into infant formula supports a sufficient supply of essential amino acids including Trp in FF infants [26, 27]. Studies on increased ALAB contents in formulae have shown $11-57 \mathrm{kcal} /$ day lower energy intakes and $0.9-1.9 \mathrm{~g} /$ day higher weight gains $[9,13,14]$. Combination of these factors increases the EE for weight by about 0.56$0.78 \mathrm{~g} / 100 \mathrm{kcal}$. Dietary ALAB is associated with higher plasma Trp concentrations $[9,14]$. Since Trp is a precursor of serotonin and melatonin, this might affect appetite regulation, satiety, and the sleep-wake rhythm [28]. If ALAB-rich formulae induce greater satiety, a lower energy intake may result. Further indication of the importance of Trp is obtained from studies which investigated the addition of free Trp to infant formulae. In the study of Hanning et al. [2], a high Trp content without an increase in the whey-to-casein ratio or the ALAB content resulted in a $63 \mathrm{kcal} /$ day lower energy intake compared to the control group. Trp is more quickly absorbed in its free form than as protein-bound amino acid and may reach tissues such as the brain or liver more rapidly [25]. The resulting increase in the ratio of Trp to the other large neutral amino acids could lead to greater satiety and less formula intake [2].

Consistent results indicate a strong relationship between a higher whey-to-casein ratio and a higher EE [12, $13,15,17]$. Whey-dominant formulae have a whey-tocasein ratio $(60: 40)$ similar to that of human milk in early lactation [29]. Whey has a slightly higher protein quality (protein digestibility-corrected amino acid score) than casein and is more easily digested by the gastrointestinal system [30]. Similar effects of higher wheyto-casein ratios on EE are observed with greater contents of intact whey $[12,13,15]$ or fully hydrolyzed whey [17].

Although the increased EE of infant formulae may be considered beneficial, infantile growth should not exceed the growth of BF infants by feeding large volumes of energetically highly efficient formulae. High growth velocities in early infancy have been related to higher adiposity and type 2 diabetes risks in later life $[4,5,8,31]$. Thus, a potential benefit for infants is derived from consuming smaller amounts of high-quality formula, while achieving adequate growth, and avoiding an additional meta- 
bolic burden. With respect to protein intake, this enables a reduction of the renal load via a lower nitrogen intake and a consequent reduction of the excretion of excess nitrogen [32].

This review and its conclusion are limited by the quality of the trials included. One of 14 trials was not a randomized controlled trial [10], and 2 of 13 studies had a very small sample size $[10,11]$. In most studies, more than one macronutrient-related factor differed between the compared formulae. Furthermore, the included studies were heterogeneous, which precluded a meta-analysis. Thus, conclusions on specific factors are limited, and this review may rather be considered hypotheses generating. Evaluation of the EE of a study is difficult when there are parallel changes in the quality and content of a nutrient. This literature review of 14 intervention trials indicates that also minor changes in the macronutrient quality or source of an infant formula have an impact on the EE for growth.

We conclude that there are indications for EE-increasing effects of an increased whey-to-casein ratio, a higher ALAB content, a higher Trp content, and im- proved fat absorption in infant formulae, while the effects of the carbohydrate source are small. These findings may contribute to explaining the differences in growth and metabolism between BF and FF infants and may be useful for developing further strategies aimed at reducing the remaining gap in metabolic processes between FF and $\mathrm{BF}$ infants.

\section{Acknowledgements}

The work reported herein was carried out with partial financial support from the Commission of the European Communities, 7th Framework Programme, contract FP7-289346-EARLY NUTRITION, and European Research Council Advanced Grant ERC2012-AdG - No. 322605 META-GROWTH. This manuscript does not necessarily reflect the views of the Commission and in no way anticipates the future policy in this area.

\section{Disclosure Statement}

None of the authors declares any conflict of interest.

\section{References}

1 Butte NF: Energy requirements of infants. Public Health Nutr 2005;8:953-967.

2 Hanning RM, Paes B, Atkinson SA: Protein metabolism and growth of term infants in response to a reduced-protein, 40:60 whey: casein formula with added tryptophan. Am J Clin Nutr 1992;56:1004-1011.

-3 Butte NF, Smith EO, Garza C: Energy utilization of breast-fed and formula-fed infants. Am J Clin Nutr 1990;51:350-358.

4 Arenz S, et al: Breast-feeding and childhood obesity - a systematic review. Int J Obes Relat Metab Disord 2004;28:1247-1256.

5 Owen CG, et al: Does breastfeeding influence risk of type 2 diabetes in later life? A quantitative analysis of published evidence. Am J Clin Nutr 2006;84:1043-1054.

6 Koletzko B, et al: Lower protein in infant formula is associated with lower weight up to age 2 years: a randomized clinical trial. Am J Clin Nutr 2009;89:1836-1845.

7 Koletzko B, et al: Infant feeding and later obesity risk. Adv Exp Med Biol 2009;646:15-29.

8 Weber $\mathrm{M}$, et al: Lower protein content in infant formula reduces BMI and obesity risk at school age: follow-up of a randomized trial. Am J Clin Nutr 2014;99:1041-1051.

-9 Fleddermann M, et al: Infant formula composition affects energetic efficiency for growth: the BeMIM study, a randomized controlled trial. Clin Nutr 2014;33:588-595.
10 Fomon SJ, et al: What is the safe protein-energy ratio for infant formulas? Am J Clin Nutr 1995;62:358-363.

11 Carnielli VP, et al: Structural position and amount of palmitic acid in infant formulas: effects on fat, fatty acid, and mineral balance. J Pediatr Gastroenterol Nutr 1996;23:553560.

12 Turck D, et al: Adequacy and safety of an infant formula with a protein/energy ratio of 1.8 $\mathrm{g} / 100 \mathrm{kcal}$ and enhanced protein efficiency for term infants during the first 4 months of life. J Pediatr Gastroenterol Nutr 2006;43: 364-371.

13 Rozé JC, et al: An alpha-lactalbumin-enriched and symbiotic-supplemented v. a standard infant formula: a multicentre, double-blind, randomised trial. Br J Nutr 2012;107:16161622.

14 Davis AM, et al: Alpha-lactalbumin-rich infant formula fed to healthy term infants in a multicenter study: plasma essential amino acids and gastrointestinal tolerance. Eur J Clin Nutr 2008;62:1294-1301.

15 Räihä NC, et al: Whey predominant, whey modified infant formula with protein/energy ratio of $1.8 \mathrm{~g} / 100 \mathrm{kcal}$ : adequate and safe for term infants from birth to four months. J Pediatr Gastroenterol Nutr 2002;35:275-281.
16 Vandenplas $\mathrm{Y}$, et al: The nutritional value of a whey hydrolysate formula compared with a whey-predominant formula in healthy infants. J Pediatr Gastroenterol Nutr 1993;17: 92-96.

17 Schmelzle H, et al: Randomized double-blind study of the nutritional efficacy and bifidogenicity of a new infant formula containing partially hydrolyzed protein, a high beta-palmitic acid level, and nondigestible oligosaccharides. J Pediatr Gastroenterol Nutr 2003;36: 343-351.

18 Heubi J, et al: Randomized multicenter trial documenting the efficacy and safety of a lactose-free and a lactose-containing formula for term infants. J Am Diet Assoc 2000;100:212217.

19 Lasekan JB, et al: Lactose-free milk proteinbased infant formula: impact on growth and gastrointestinal tolerance in infants. Clin Pediatr 2011;50:330-337.

20 Koo WW, et al: Reduced bone mineralization in infants fed palm olein-containing formula: a randomized, double-blinded, prospective trial. Pediatrics 2003;111:1017-1023.

21 Kennedy K, et al: Double-blind, randomized trial of a synthetic triacylglycerol in formulafed term infants: effects on stool biochemistry, stool characteristics, and bone mineralization. Am J Clin Nutr 1999;70:920-927. 
22 Ludwig DS: The glycemic index: physiological mechanisms relating to obesity, diabetes, and cardiovascular disease. JAMA 2002;287: 2414-2423.

23 Koletzko B, et al: Protein intake in the first year of life: a risk factor for later obesity? The E.U. childhood obesity project. Adv Exp Med Biol 2005;569:69-79.

24 Sala-Vila A, et al: Lipid composition in human breast milk from Granada (Spain): changes during lactation. Nutrition 2005;21: 467-473.
25 Lonnerdal B: Infant formula and infant nutrition: bioactive proteins of human milk and implications for composition of infant formulas. Am J Clin Nutr 2014;99:22.

26 Lonnerdal B, Lien EL: Nutritional and physiologic significance of alpha-lactalbumin in infants. Nutr Rev 2003;61:295-305.

27 Wurtman RJ, Wurtman JJ: Do carbohydrates affect food intake via neurotransmitter activity? Appetite 1988;1:42-47.

28 Lien EL: Infant formulas with increased concentrations of alpha-lactalbumin. Am J Clin Nutr 2003;77:1555S-1558S.

29 Lonnerdal B: Nutritional and physiologic significance of human milk proteins. Am J Clin Nutr 2003;77:1537S-1543S.
30 Molgaard C, et al: Milk and growth in children: effects of whey and casein. Nestle Nutr Workshop Ser Pediatr Program 2011;67:6778.

31 Brands B, Demmelmair H, Koletzko B: How growth due to infant nutrition influences obesity and later disease risk. Acta Paediatr 2014; 103:578-585.

32 Axelsson I: Effects of high protein intakes. Nestle Nutr Workshop Ser Pediatr Program 2006;58:121-129. 